\title{
Patrones de distribución del complejo “Myotis mystacinus” en la península Ibérica
}

\author{
Jesús Nogueras*, José Antonio Garrido-García, Alberto Fijo-León, \\ JaVier Juste, Juan Luis García-Mudarra, CARlos IbáÑEZ \\ Estación Biológica de Doñana (CSIC), Apartado 1056, 41080 Sevilla (Spain) \\ * Correo electrónico del autor: nogueras@ebd.csic.es \\ DOI: http://dx.doi.org/10.14709/BarbJ.6.1.2013.03
}

English title: Distribution patterns of the "Myotis mystacinus" complex in the Iberian Peninsula

\begin{abstract}
We present the first record of whiskered bat (Myotis mystacinus) for Andalusia (Spain) from the Sierra de Huéscar, in Granada and discuss the distribution pattern of the "M. mystacinus" complex in Iberia with the recent description of its sibling species M. alcathoe. Whereas M. mystacinus is present across the whole distribution area known for the species complex, it seems that -according to the present sampling information- the species M. alacathoe is really not found in the Central Pyrenees, Sistema Central, Sistema Ibérico Sur and Sierras Béticas mountain massifs. Despite being sympatric in several localities, M. mystacinus reaches higher altitudes than M. alcathoe.
\end{abstract}

Keywords: Myotis mystacinus, distribution patterns, Iberian Peninsula.

Resumen: Se cita por primera vez Myotis mystacinus en Andalucía en la Sierra de Huéscar (Granada) y se revisa la distribución en Iberia del complejo $M$. mystacinus tras la reciente descripción de $M$. alcathoe. Myotis mystacinus ocupa toda el área de distribución conocida para el complejo, mientras que la ausencia de M. alcathoe en el Pirineo Central, Sistema Central, Sistema Ibérico Sur y Sierras Béticas parece ser real, en base al nivel de muestreo disponible en la actualidad. A pesar de la coexistencia de las especies en distintas localidades, se aprecian diferencias en sus respectivas distribuciones altitudinales, alcanzando M. mystacinus cotas más elevadas.

Palabras clave: Myotis mystacinus, patrones de distribución, Península Ibérica.

\section{INTRODUCCIÓN}

El murciélago ratonero de Alcathoe (Myotis alcathoe) es una especie morfológicamente muy similar al murciélago ratonero bigotudo $(M$. mystacinus) de amplia distribución en Europa. De hecho, la especie ha sido descrita muy recientemente a partir de técnicas moleculares aunque también se encontraron algunas diferencias en cariotipo, morfología y ecolocación en relación al ratonero bigotudo (Helversen et al. 2001).
La descripción se realizó con ejemplares de Grecia, lo que en un principio llevó a pensar que podía tratarse de un endemismo balcánico, pero se ha ido encontrando progresivamente en numerosos países europeos: Francia (Ruedi et al. 2002), Eslovaquia (Benda et al. 2003), España (AgirreMendi et al. 2004), Bulgaria (Schunger et al. 2004), Suiza (Stadelmann et al. 2004), Alemania, Polonia, Albania y Turquía (Niermann et al. 2007), Austria (Spitzenberger 2008), Republica Checa (Lucan et al. 2009), Inglaterra (Jan et al. 2010), Italia (Galimberti et al. 2012) y Croacia (Pavlinic 2012). Por lo tanto la especie está presente en 
gran parte de Europa y muestra un importante solapamiento con la distribución de su especie gemela M. mystacinus.

Debido a esta circunstancia en las áreas en las que están presentes ambas especies la información anterior a la descripción de M. alcathoe en 2001, solo puede asignarse de forma genérica al complejo "M. mystacinus" o M. mystacinus s.l. La principal consecuencia es que no están bien definidos los patrones de distribución de ambas especies, ni a nivel general, ni local.

En este trabajo aportamos información original que amplia significativamente la distribución de $M$. mystacinus en la península Ibérica con ejemplares de Andalucía. Además tratamos de definir los patrones de distribución de ambas especies en la península Ibérica a partir de la información acumulada en los últimos años.

\section{Material Y MÉtodos}

Para el estudio comparado de los patrones de distribución, nos hemos basado en la información bibliográfica que la hemos agrupamos de dos maneras, por una parte los artículos recientes en los que se mencionan ejemplares identificados molecularmente, procedentes de La Rioja (Agirre-Mendi et al. 2004), Galicia (Hermida et al. 2007), Aragón (Alcalde et al. 2008), Navarra (Alcalde 2009), Cataluña (Flaquer et al. 2010), Portugal (Barros et al. 2012). Por otra parte todo lo publicado antes de la diferenciación de ambas especies y que consideramos como perteneciente al complejo " $M$. mystacinus" o M. mystacinus s.l. Esta última información se ha extraído básicamente de la edición de 2007 del Atlas de los mamíferos españoles (Palomo et al. 2007).

Además, aportamos una nueva cita a partir de datos propios inéditos procedentes de los trabajos de inventariación de murciélagos forestales en Andalucía en el marco del proyecto: "Sistemas de gestión de las poblaciones de murciélagos forestales de Andalucía", realizado entre los años de 2009 y 2011 y cuyo principal objetivo era mejorar el conocimiento sobre los murciélagos forestales en Andalucía a través del estudio de la distribución de las diferentes especies, delimitación de las zonas más importantes para este grupo en la región y la identificación de los principales problemas de conservación (Ibáñez et al. 2012). Para este estudio se han prospectado un total de 244 localidades (124 cuadrículas $10 \times 10 \mathrm{~km}$ ) distribuidas por toda la Comunidad Autónoma y repartidas por las formaciones boscosas más importantes (Fig. 1). En las 200 sesiones de muestreo realizadas durante el proyecto (2009-2011), se han invertido un total de 860 horas, utilizando redes de niebla con una cobertura total de 4713 metros de red colocadas en el periodo de junio - agosto sobre posibles bebederos. En el estudio se ha capturado un total de 2675 ejemplares de murciélagos de 21 especies diferentes.

La identificación molecular de la totalidad de los ejemplares (tanto los relacionados en las publicaciones mencionadas, como los inéditos) se ha realizado por los autores en el Laboratorio de Ecología Molecular de la Estación Biológica de Doñana (CSIC). Del ADN extraído de biopsias alares, se amplificó un fragmento mitocondrial de aproximadamente 600 pares de bases del gen de la subunidad 1 del complejo enzimático NADH deshidrogenasa (ND1) según se detalla en GarcíaMudarra et al. (2009).

\section{RESULTADOS Y DISCUSIÓN}

\section{El murciélago ratonero bigotudo (Myotis mystacinus) en Andalucía}

Durante los muestreos del proyecto forestal se capturaron el 16/07/2009 4 ejemplares (todas hembras lactantes) de M. mystacinus mediante redes de niebla en dos localidades diferentes del norte de la provincia de Granada (Huéscar y Puebla de Don Fabrique, en la Sierra de Huéscar), apenas distantes entre sí $4 \mathrm{~km}$ que suponen dos nuevas cuadrículas $10 \times 10 \mathrm{~km}$ para la distribución de la especie y la primera cita para la región.

Las localidades de capturas son dos puntos de agua, entre los 1450 y 1550 m s.n.m., utilizados como bebederos. Uno estaba situado en un pequeño manantial al inicio de un barranco bastante cerrado $y$ el otro en una alberca utilizada para abastecer al ganado, ambas situadas en bosque maduro y de gran porte de Pinus nigra, con ejemplares viejos dispersos de Quercus faginea.

En ambos muestreos se capturaron 81 murciélagos de al menos 11 especies diferentes: Myotis emarginatus (2), Myotis escalerai (4), Myotis bechsteinii (1), Myotis mystacinus (4), Myotis myotis (3), Barbastella barbastellus (4), = Miniopterus schreibersii (4), Plecotus austriacus (10), Hypsugo savii (20), Pipistrellus pipistrellus / P. pygmaeus (25), Pipistrellus kuhlii (4). 
A

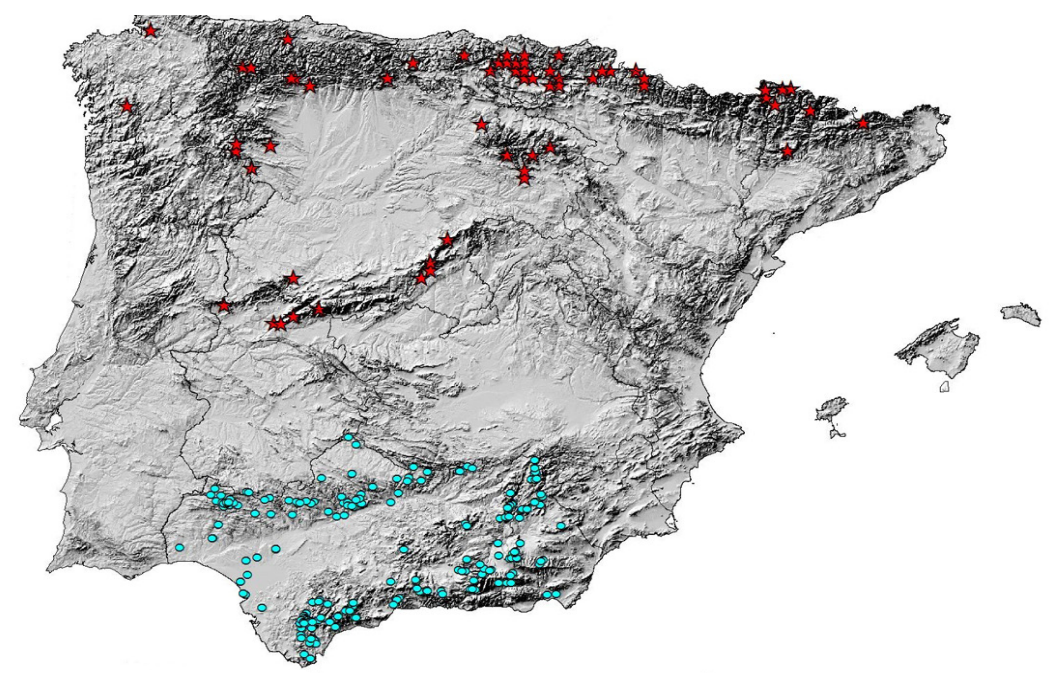

B

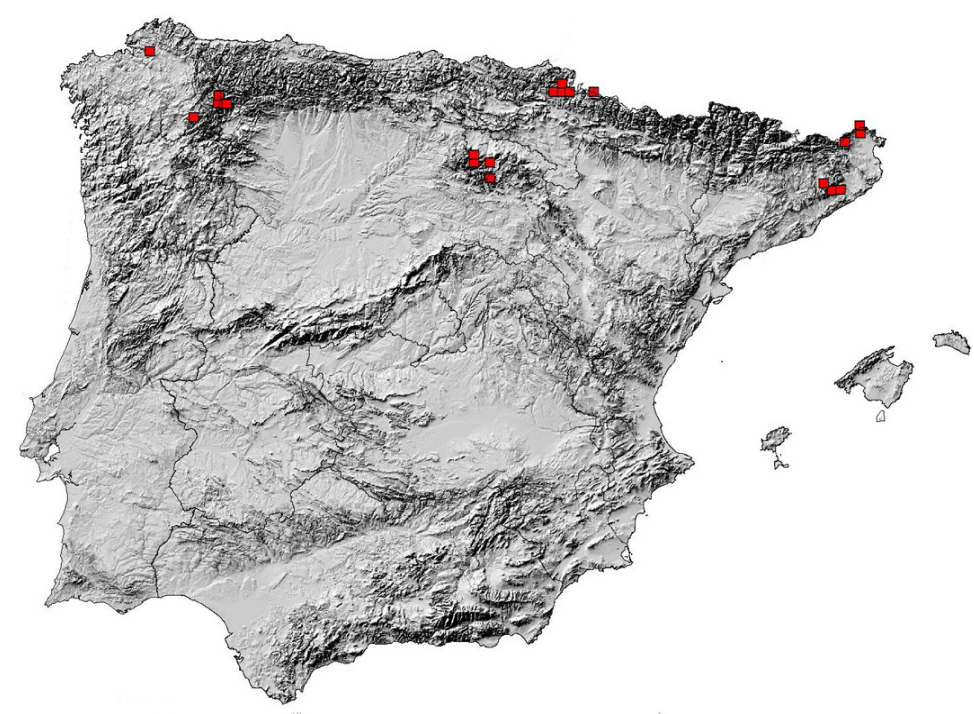

$\mathrm{C}$

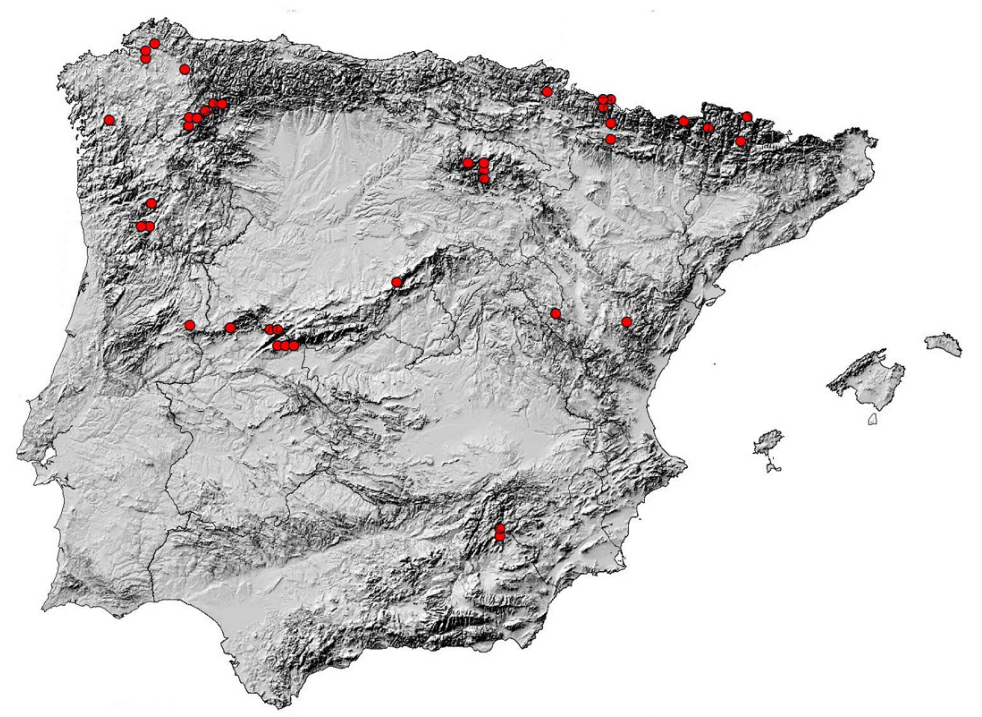

Fig. 1 Distribución del complejo "Myotis mystacinus" en la Península Ibérica (cuadrículas 10X10 km): A.- estrellas Myotis mystacinus s.I, círculos, localidades muestreadas en Andalucía. B.cuadrados M. alcathoe confirmados. C.- círculos M. mystacinus confirmados. Citas de acuerdo a Agirre-Mendi et al. 2004, Alcalde et al. 2008, Alcalde 2009, Barros et al. 2012, Flaquer et al. 2010, Hermida et al. 2007, Palomo et al. 2007, Schreur 2007 y datos propios inéditos. 
Tabla 1: Relación de cuadrículas con presencia del complejo "M. mystacinus". Se proporcionan las cuadrículas $10 X 10 \mathrm{~km}$ con identificación segura para cada especie

\begin{tabular}{|c|c|c|c|c|c|c|c|c|c|c|c|c|c|c|c|}
\hline UTM10 & $\frac{\frac{U}{\pi}}{\sum}$ & $\stackrel{\hat{\Xi}}{\sum}$ & $\begin{array}{l}\overrightarrow{\dot{n}} \\
\hat{\hat{E}} \\
\dot{\mathrm{E}}\end{array}$ & UTM10 & $\frac{\frac{U}{\sigma}}{\Sigma}$ & 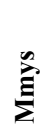 & $\begin{array}{l}\overline{\dot{s}} \\
\hat{a} \\
\dot{\Xi}\end{array}$ & UTM10 & $\frac{\frac{U}{\pi}}{\sum}$ & 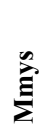 & $\begin{array}{l}\vec{n} \\
\hat{a} \\
\dot{\Xi}\end{array}$ & UTM10 & $\frac{0}{\pi}$ & 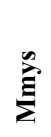 & $\begin{array}{l}\overline{\dot{n}} \\
\hat{\vec{A}} \\
\dot{\mathrm{z}}\end{array}$ \\
\hline 29TNF98 & & $\mathrm{X}$ & & 29TQH45 & & & $\mathrm{X}$ & 30TWM22 & & & $X$ & 30TXN64 & & & $\mathrm{X}$ \\
\hline 29TNH41 & & $\mathrm{X}$ & & 29TQJ30 & & & $\mathrm{X}$ & 30TWM23 & & & $X$ & $30 \mathrm{TXN} 65$ & & & $\mathrm{X}$ \\
\hline 29TNH50 & & & $\mathrm{X}$ & 30SWH40 & & $\mathrm{X}$ & & 30TWM25 & $X$ & $\mathrm{X}$ & & 30TXN74 & & $X$ & \\
\hline 29TNH89 & & $\mathrm{X}$ & & 30SWH41 & & $\mathrm{X}$ & & 30TWM26 & & $\mathrm{X}$ & & $30 \mathrm{TXN75}$ & & $\mathrm{X}$ & \\
\hline 29TNJ70 & & & $\mathrm{X}$ & 30TTK54 & & & $\mathrm{X}$ & 30TWM27 & $\mathrm{X}$ & $\mathrm{X}$ & & $30 \mathrm{TXN} 80$ & & $\mathrm{X}$ & \\
\hline 29TNJ80 & $\mathbf{X}$ & $\mathrm{X}$ & & 30TTK56 & & $X$ & & 30TWM35 & & & $X$ & $30 \mathrm{TXN} 82$ & & $\mathrm{X}$ & \\
\hline 29TNJ91 & & $\mathrm{X}$ & & 30TTK59 & & & $\mathrm{X}$ & 30TWM56 & & & $X$ & $30 \mathrm{TXN} 85$ & & $\mathrm{X}$ & \\
\hline 29TPF08 & & $\mathrm{X}$ & & 30TTK64 & & $\mathrm{X}$ & & 30TWN07 & & & $\mathrm{X}$ & 30TYK07 & & $\mathrm{X}$ & \\
\hline 29TPE66 & & $\mathrm{X}$ & & 30TTK66 & & $\mathrm{X}$ & & 30TWN08 & & & $\mathrm{X}$ & 31TBH82 & & $\mathrm{X}$ & \\
\hline 29TPE85 & & & $\mathrm{X}$ & 30TTK74 & & $\mathrm{X}$ & & 30TWN16 & & & $X$ & 31 TCG35 & & & $\mathrm{X}$ \\
\hline 29TPG01 & & $\mathrm{X}$ & & 30TTK84 & & $X$ & & 30TWN17 & & & $X$ & 31TCG59 & & $\mathrm{X}$ & \\
\hline 29TPG85 & & & $\mathrm{X}$ & 30TTK85 & & & $\mathrm{X}$ & 30TWN25 & & & $\mathrm{X}$ & $31 \mathrm{TCH} 11$ & & $\mathrm{X}$ & \\
\hline 29TPG86 & & & $\mathrm{X}$ & 30TTN55 & & & $\mathrm{X}$ & 30 TWN26 & & & $\mathrm{X}$ & $31 \mathrm{TCH} 12$ & & & $\mathrm{X}$ \\
\hline 29TPH38 & & $\mathrm{X}$ & & 30TTN74 & & & $\mathrm{X}$ & 30TWN27 & & & $\mathrm{X}$ & $31 \mathrm{TCH} 13$ & & & $\mathrm{X}$ \\
\hline 29TPH41 & & $\mathrm{X}$ & & 30TUN65 & & & $\mathrm{X}$ & 30TWN28 & & & $\mathrm{X}$ & $31 \mathrm{TCH} 21$ & & & $\mathrm{X}$ \\
\hline 29TPH42 & $\mathbf{X}$ & $\mathrm{X}$ & & 30TUN97 & & & $\mathrm{X}$ & 30TWN35 & & & $X$ & $31 \mathrm{TCH} 33$ & & & $\mathrm{X}$ \\
\hline 29TPH52 & & $\mathrm{X}$ & & 30TVK09 & & & $\mathrm{X}$ & 30TWN54 & & & $\mathrm{X}$ & $31 \mathrm{TCH} 43$ & & & $X$ \\
\hline 29TPH63 & & $\mathrm{X}$ & & 30TVL10 & & & $\mathrm{X}$ & 30TWN56 & & & $\mathrm{X}$ & $31 \mathrm{TCH} 60$ & & & $\mathrm{X}$ \\
\hline 29ТРН74 & $\mathbf{X}$ & $X$ & & 30TVL11 & & & $\mathrm{X}$ & 30TWN64 & & & $X$ & 31TCH62 & & $\mathrm{X}$ & \\
\hline 29TPH75 & $\mathbf{X}$ & & & 30TVL12 & & $\mathrm{X}$ & & 30TWN65 & & & $\mathrm{X}$ & 31TDG28 & & & $\mathrm{X}$ \\
\hline 29TPH84 & $\mathbf{X}$ & $X$ & & 30TVL34 & & & $\mathrm{X}$ & 30TWN68 & & & $X$ & 31TDG43 & $\mathrm{X}$ & & \\
\hline 29TPH86 & & & $\mathrm{X}$ & 30TVM79 & & & $\mathrm{X}$ & 30TXK18 & & $\mathrm{X}$ & & 31TDG52 & $\mathrm{X}$ & & \\
\hline 29TPH96 & & & $X$ & 30 TVN58 & & & $\mathrm{X}$ & $30 \mathrm{TXN} 05$ & & & $\mathrm{X}$ & 31TDG62 & $\mathrm{X}$ & & \\
\hline 29TQE16 & & $X$ & & 30 TVN86 & & & $\mathrm{X}$ & $30 \mathrm{TXN} 06$ & $\mathrm{X}$ & $\mathrm{X}$ & & 31TDG78 & $\mathrm{X}$ & & \\
\hline 29TQE43 & & & $\mathrm{X}$ & 30TVN97 & & & $\mathrm{X}$ & $30 \mathrm{TXN} 16$ & $\mathrm{X}$ & & $\mathrm{X}$ & 31TDG99 & $\mathrm{X}$ & & \\
\hline 29TQE53 & & & $\mathrm{X}$ & 30TWM05 & & & $\mathrm{X}$ & $30 \mathrm{TXN} 17$ & $\mathrm{X}$ & & & $31 \mathrm{TDH} 90$ & $\mathrm{X}$ & & \\
\hline 29TQG03 & & & $X$ & 30TWM07 & $\mathrm{X}$ & $\mathrm{X}$ & & $30 \mathrm{TXN} 26$ & $\mathrm{X}$ & & $X$ & & & & \\
\hline 29TQG26 & & & $\mathrm{X}$ & 30TWM08 & $\mathrm{X}$ & & & $30 \mathrm{TXN} 56$ & $\mathrm{X}$ & & $\mathrm{X}$ & & & & \\
\hline
\end{tabular}

Las 4 hembras presentaban pelaje dorsal pardo rojizo y la base interna de la oreja y el trago de color oscuro. La medida del antebrazo es $34,8 \pm 0,5$ $\mathrm{mm}(\mathrm{n}=3)$. Las secuencias del fragmento de ND1 de estos individuos presentan una similitud del 99\% con las de los individuos de M. mystacinus ibéricos ya publicadas (García-Mudarra et al. 2009), validando su pertenencia al mismo linaje.

Se trata de una especie con un fuerte carácter forestal, localizándose en zonas montañosas de bosques maduros de frondosas y coníferas. Resulta difícil su captura en espacios abiertos o balsas incluidas en el bosque, resultando más eficaz los arroyos de gargantas encajadas con abundante vegetación de ribera y ambiente húmedo y fresco. Aunque su captura resulta ser poco frecuente, en parte debido a la especificidad del hábitat que frecuenta, allí donde se encuentra se suele capturar.

En base a los datos de intensidad de muestreo y superficie prospectada, y que solo se ha encontrado en las localidades mencionadas, se puede considerar muy probable que esta especie no se localice en ningún otro sitio de Andalucía fuera del macizo de Cazorla, Segura, Castril - Huéscar y Alcaraz (Jaén, Granada y Albacete), dado la continuidad del sistema montañoso, la climatología y la cubierta forestal. El rango altitudinal en el que se ha encontrado en su distribución andaluza, es otro factor a tener en cuenta en el diseño de futuras prospecciones.

Las localidades más próximas de la Península Ibérica en donde era conocido M. mystacinus se sitúan en el Sistema Central y Sistema Ibérico Sur. La nueva localidad que se aporta para Andalucía, supone una ampliación de dicha distribución hacia el sur de unos 300 y $265 \mathrm{~km}$ respectivamente. Hay que señalar que la especie es conocida en Marruecos por lo que esta nueva localidad no es especialmente sorprendente. 
Tabla 2 Zonificación del área de distribución del complejo "Myotis mystacinus". Se proporciona el número de individuos identificados molecularmente y el porcentaje que representa sobre el número de muestras secuenciadas.

\begin{tabular}{|c|c|c|c|c|c|c|}
\hline \multicolumn{2}{|l|}{ REGIONES } & \multirow{2}{*}{$\hat{\Sigma}_{32}^{\infty}$} & \multirow{2}{*}{$\sum_{e^{\circ}}^{\infty}$} & \multirow{2}{*}{$\frac{\sum^{\frac{0}{J}}}{12}$} & \multirow{2}{*}{$\frac{\sum_{\text {ê }}^{\frac{e}{J}}}{27 \%}$} & \multirow{2}{*}{$\begin{array}{l}\text { बేँี } \\
44\end{array}$} \\
\hline NO Iberia & Galicia, N Portugal, Zamora, León & & & & & \\
\hline Cornisa Cantábrica & Asturias, Cantabria, País Vasco & 0 & 0 & 0 & 0 & 0 \\
\hline Pirineo Occidental & Navarra & 6 & $27 \%$ & 16 & $73 \%$ & 22 \\
\hline Pirineo Central & Huesca, Lérida & 19 & $100 \%$ & 0 & 0 & 19 \\
\hline Pirineo Oriental & Barcelona, Gerona & 0 & 0 & 14 & $100 \%$ & 14 \\
\hline Sistema ibérico Norte & La Rioja, Burgos, Soria & 6 & $46 \%$ & 7 & $54 \%$ & 13 \\
\hline Sistema Ibérico Sur & Teruel & 7 & $100 \%$ & 0 & 0 & 7 \\
\hline Sistema Central & Madrid, Segovia, Guadalajara, Salamanca, Cáceres, Centro Portugal & 15 & $100 \%$ & 0 & 0 & 15 \\
\hline Béticas & Granada, Jaén, Albacete & 3 & $100 \%$ & 0 & 0 & 3 \\
\hline & TOTAL & 88 & & 49 & & 137 \\
\hline
\end{tabular}

Distribución de M. mystacinus y M. alcathoe en Iberia

Myotis mystacinus s.l. tiene una distribución en Iberia fragmentada y restringida a la mitad norte principalmente a sistemas montañosos. Ocupa Pirineos, Cornisa Cantábrica, Galicia y norte de Portugal, Sistema Ibérico Norte y Sur, Sistema Central (incluyendo parte de Portugal) y Sierra de Huéscar en los Sistemas Béticos en donde no había citada hasta ahora. El complejo se ha encontrado en 110 cuadrículas de 10 x $10 \mathrm{~km}$ (Tabla 1, Fig. 1).

Utilizando las citas comprobadas molecularmente, $M$. alcathoe se ha encontrado hasta el momento sólo en 20 cuadrículas distribuidas por Galicia, extremos Occidental y Oriental de Pirineos y Sistema Ibérico Norte (Tabla 1, Fig. 1). Utilizando el mismo criterio de identificación molecular, M. mystacinus se ha confirmado en 40 cuadrículas en Galicia, norte de Portugal, zona central de Pirineos, Sistemas Ibéricos Norte y Sur, Sistema Central y Sierra de Huéscar (Tabla 1, Fig. $1)$.

Para contrastar la robustez de estos patrones, hemos dividido el área de distribución del complejo "M. mystacinus" en 9 zonas: NO ibérico (Galicia y norte de Portugal), Cornisa Cantábrica, Pirineo Occidental (Navarra), Pirineo Central (Huesca y Lleida), Pirineo Oriental (Girona y Barcelona), Sistema Ibérico Norte, Sistema Ibérico Sur, Sistema Central y Sierras Béticas. Para cada una de estas zonas hemos calculado el número de individuos que han sido identificados molecularmente y el porcentaje al que pertenece cada especie (Tabla 2).

En las zonas en donde se han encontrado las dos especies, la más rara supone al menos el $20 \%$ de las muestras (Tabla 2), incluso con tamaños de muestra reducidos como por ejemplo en el Sistema Ibérico Norte (13).

De acuerdo con estos resultados, el nivel de muestreo disponible en general es suficiente para señalar que M. mystacinus ocupa toda o casi toda el área conocida previamente del complejo " $M$. mystacinus" (queda por confirmar su presencia en la Cornisa Cantábrica), faltando o siendo muy escaso solo en el Pirineo Oriental y sus estribaciones. Myotis alcathoe por el contrario parece mostrar una distribución más restringida en el norte peninsular. Su ausencia en las Sierras Béticas, Sistema Ibérico Sur, Sistema Central y Pirineo Central parece que es real y no producto de falta de muestreo. Como en el caso de M. mystacinus es necesario muestrear en la Cornisa Cantábrica para confirmar su presencia.

Aunque ambas especies conviven en diferentes localidades (en 8 cuadrículas coexisten ambas especies), los rangos altitudinales presentan diferencias apreciables y mientras M. alcathoe se localiza a una altitud media de $529 \mathrm{~m}$ (140 - 1391 m. s.n.m, n=44), M. mystacinus se localiza más frecuentemente a mayores altitudes $(\bar{X}=1116$; 350-1965 m s.n.m, $n=70$ ) 


\section{REFERENCIAS}

Aguirre-Mendi, P.T., García-Mudarra, J.L., Juste, J. \& IbÁÑEZ, C. 2004. Presence of Myotis alcathoe Helversen \& Heller, 2001 (Chiroptera: Vespertilionidae) in the Iberian Peninsula. Acta Chiropterologica 6: 49-57. DOI: $\underline{\text { https://dx.doi. }}$ org/10.3161/1508110042176590

AlCalde, J.T. 2009. Myotis alcathoe Helversen \& Heller, 2001 y Pipistrellus pygmaeus (Leach, 1825), nuevas especies de quirópteros para Navarra. Munibe 57: 225-236.

Alcalde, J.T. Trujillo, D., Artázcoz, A., \& AgirRe-Mendi P.T., 2008. Distribución y estado de conservación de los quirópteros de Aragón. Graellsia 64: 3-16.

BArros, P. 2012. Contribución al conocimiento de la distribución de quirópteros en el norte y centro de Portugal. Barbastella 5: 19-31. DOI: http://dx.doi.org/10.14709/ BarbJ.5.1.2012.04

Benda, P., Ruedi, M., \& Uhrin, M. 2003. First record of Myotis alcathoe (Chiroptera: Vesper tilionidae) in Slovakia. Folia Zoologica 52: 359-365.

Flaquer, C., Puig, X., Fabregas, E., Guixé, D., Torre, I., Rafols R.G., PÁramo, F., Camprodon, J., Cumplido, J.Mº., RuízJarillo, R., Baucells, A.L., Freixas, L., \& Arrizabalaga, A. 2010. Revisión y aportación de datos sobre quirópteros de Cataluña: propuesta de lista roja. Galemys 22: 29-61.

Galimberti, A., Spada, M., Russo, D., Mucedda, M., Agnelli, P., Ferrí, E., Martinoli, A., \& CASIRAGHI, M. 2012. Integrated operational taxonomic units (IOTUs) in echolocating bats: a bridge between molecular and traditional taxonomy. Plos ONE 7(6): e40122. DOI: https://dx.doi.org/10.1371/ journal.pone.0040122

García-Mudarra, J.L. IbÁÑEZ, C. \& Juste, J., 2009. The strait of Gibraltar; barrier or bridge to the Ibero-Moroccan bat diversity?. Biological Journal of the Linnean Society 96: 434-450. DOI: $\quad$ http://dx.doi.org/10.1111/j.10958312.2008.01128.x
Helversen, O., Heller, K.-G F., Mayer, F., Nemeth, A., Volleth, M. \& Gombкötö. P. 2001. Cryptic mammalian species: a new species of whiskered bat (Myotis alcathoe n. sp.) in Europe. Naturwissenschaften 88: 217-223. DOI: https://dx.doi.org/10.1007/ $\underline{\mathrm{s} 001140100225}$

Hermida, R.J., Lamas,F.J., Graña, D. A., Rial, S., Cerqueira, F., Arzúa, M., \& Seage, R. 2012. Contribución al conocimiento de los Murciélagos (O. Chiroptera) en Galicia. Galemys 24, 13-23. DOI: https://dx.doi. org/10.7325/Galemys.2012.A02

IbáÑez, C., Nogueras, J., Puig, X., Juste, J. \& Schreur, G. 2012. Sistemas de gestión de las poblaciones de murciélagos forestales en Andalucía. Informe final inédito. Estación Biológica de Doñana (CSIC) / Consejería de Medio Ambiente de la Junta de Andalucía. $165 \mathrm{pp}$

Jan, M. I., Frith, K., Glover, A.M., Butlin, K., Scott, F., Ruedi, M., Frantz, A.C. Dawson, D.A. \& Altringham, J.D. 2010. Myotis alcathoe confirmed in the UK from mitochondrial and microsatellite DNA. Acta Chiropterologica 12: 471-483. DOI: http:// dx.doi.org/10.3161/150811010X538043

LuCAN, R.K.,ANDREAS, M.,BENDA,P.,BARTONICKA, T., Brezinová, T., Hoffmannová, A., Hulova, S., Hulva, P., Neckarova, J., Reiter, A., Svacina, T., SaleK, M., \& HoraceK, I. 2009. Alcathoe bat (Myotis alcathoe) in the Czech Republic: distributinal status, roosting and feeding ecology. Acta Chiropterologica 11: 60-69. DOI: http:// dx.doi.org/10.3161/150811009X465695

NiERmann,I.,Biedermann,M.,Bogdanowicz,W., Brinkmann, R., Lebris, Y., Ciechanowski, M., Dietz, C., Dietz, I., EstóK, P., Helversen, O. Von, Le Houńdec, A., Paksuz, S., Petrov, B.P., ÖZKan, B., PIKSA, K., RaChWald, A., RouÉ,S.Y.,SachanowicZ,K.,SCHORCht,W., TerebA, A., \& MAYER, F. 2007. Biogeography of the recently described Myotis alcathoe von Helversen and Heller, 2001. Acta Chiropterologica 9: 361- 378. 
Palomo, L. J., Gisbert, J. \& Blanco. J. C. 2007. Atlas y Libro Rojo de los Mamíferos Terrestres de España. Dirección General para la Biodiversidad -SECEM- SECEMU, Madrid. 588 pp.

Ruedi, M., Jourde, P., Giosa, P., Barataud, M., \& RouÉ, S. Y. 2002. DNA reveals the existence of Myotis alcathoe in France (Chiroptera: Vespertilionidae). Revue suisse de Zoologie 109: 643-652.

Schreur, G. 2007. Seguimiento de quirópteros forestales en Extremadura. Proyecto LIFE - Naturaleza "Conservación de quirópteros amenazados en Extremadura" Consejería de Agricultura y Medio Ambiente. Junta de Extremadura - SECEMU 2007.
Schunger, I., Dietz, C., Merdschanova, D., Merdschanov, S., Christov, K., Borissov, I., Staneva, S. \& Petrov, B. 2004. Swarming of bats (Chiroptera, Mammalia) in the Vodnite Dupki Cave (Central Balkan National Park, Bulgaria). Acta Zoologica Bulgarica 56: 323-330. DOI: http://dx.doi. org/10.3161/150811011X578660

Spitzenberger, F., Pavlinic, I. \& Podnar, M. 2008. On the occurrence of Myotis alcathoe von Helversen and Heller, 2001 in Austria. Hystrix It. J. Mamm. (n.s) 19: 3-12

Stadelmann, B., Jacobs, D.S., Schoeman, C. \& RuedI, M. 2004. Phylogeny of African Myotis bats (Chiroptera, Vespertilionidae) inferred from cytochrome $b$ sequences. Acta Chiropterologica 6: 177-192. DOI: http:// dx.doi.org/10.3161/001.006.0201 